\title{
Nivel de satisfacción de requerimientos \\ energéticos y de macronutrientes en \\ la ingesta usual de escolares en sectores \\ rurales de la sierra ecuatoriana
}

\author{
Level of satisfaction of energy and \\ macronutrients requirements in the \\ usual intake of kids in rural areas \\ of the ecuadorian highlands
}

\begin{abstract}
This study, conducted among students from rural areas of Pujilí Canton, Cotopaxi Province, aged 8 to 15 years, allowed to determine: 1) The individual energy requirements by levels of physical activity; 2) the levels of energy and macronutrient sufficiency and balance of diet seen in the light of international standards. It was found that more than $70 \%$ of schoolchildren do not meet the suggested range by the Joint Expert (World Health Organization / Food and Agriculture Organization of the United Nations/ United Nations University) for energy and fat intakes, but that percentage is less than $40 \%$ for proteins and carbohydrates. Also, the results indicate that the percentage contribution from usual macronutrient intake of schoolchildren to their estimated energy intake reach respective goal requirements established. Key words: Usual intake; energy requirements; macronutrients; diet balance; rural schools.
\end{abstract}

Luz Marina Pereira G.

Carrera de Lingüística,

Departamento de Ciencias Humanas y Sociales Universidad de las Fuerzas Armadas (ESPE)

Secretaría de Educación Superior, Ciencia, Tecnología e Innovación (SENESCYT), Ecuador.

Dirigir la correspondencia a: Luz Marina Pereira González E-mail: luzarayma@gmail.com

Este trabajo fue recibido el 13 de Octubre de 2015 y aceptado para ser publicado el 15 de Enero de 2016.
Es un hecho conocido que la malnutrición es una de las causas principales que puede afectar la salud especialmente de individuos en edad de crecimiento. Según la Organización Mundial de la Salud (1) la malnutrición "puede reducir la inmunidad, aumentar la vulnerabilidad a las enfermedades, alterar el desarrollo físico y mental, y reducir la productividad". La malnutrición está relacionada de forma directa con el exceso o el déficit de nutrientes en las dietas habituales de los individuos y la determinación de su incidencia permite a los estados tomar las correcciones necesarias en la búsqueda de mejores condiciones de vida para la población. Para poder determinar la prevalencia de estas ingestas inadecuadas, se debería tener información sobre el consumo de alimentos lo que no siempre está disponible y cuyo levantamiento implicaría, por una parte, altos costos y, por la otra, requeriría de la disposición de las personas involucradas a formar parte de un estudio durante largo tiempo.

Una de las técnicas más usadas para obtener información sobre el consumo de alimentos en una población suele ser la Encuesta de Recordatorio de 24 Horas $(2,3,4,5)$. Según Carriquiry (6), la varianza de la distribución promedio de los datos obtenidos en dos mediciones, por ejemplo, no sólo refleja la variabilidad de individuo a individuo en la ingesta (varianza interpersonal), sino que al mismo tiempo da cuenta de las variaciones que ocurren en la ingesta de un mismo individuo entre un día y otro (varianza intraindividual).

El National Research Council (7) fue el primero en proponer la necesidad de ajustar los datos de las ingestas de alimentos para tratar de estimar, a través de métodos estadísticos, cuál es la distribución de la ingesta habitual de un individuo sobre la base de la información de algunas mediciones. Este concepto ha sido implementado por la lowa State University a través del software PC_SIDE (Software for Intake Distribution Estimation, Software para de estimación de la Distribución de la Ingesta) (8) un modelo estadístico que posee buenas propiedades (9), y sólo requiere tener registros de más de una encuesta de Recordatorio de 24 horas.

EI PC_SIDE permite estimar la varianza de la ingesta individual, que corresponde a la varianza interpersonal, y la varianza de los errores de la medición que refleja, día a día, la variación de la ingesta en un individuo (intrapersonal) $(10,11)$. El software realiza una transformación de los datos a una escala normal y luego de que es eliminada la variación intrapersonal de las medidas observadas, la ingesta usual esti- 
mada se transforma de nuevo a la escala original. La principal bondad del uso del PC_SIDE radica en que permite obtener la ingesta habitual tanto de los nutrientes como de los alimentos y la posibilidad de tomar en cuenta, al momento de realizar la estimación, variables como la edad, el sexo, el peso de los individuos y la secuencia de la medición a la cual corresponde el consumo de alimentos cuyos datos han sido obtenidos a través del test de 24 horas.

Una vez que estima la distribución de la ingesta usual, si se dispone de los requerimientos individuales de los nutrientes de interés, se puede obtener la prevalencia del consumo inadecuado como el porcentaje de individuos cuya ingesta usual no alcanza a cubrir el requerimiento estimado promedio de un nutriente en particular (EAR, Estimated Average Requirement) (12). Sin embargo, no siempre es posible disponer de la distribución de requerimientos individuales; ya que dicho cálculo requiere conocer una cantidad importante de información a nivel personal.

En el Ecuador, a través del Ministerio de Salud Pública y del Instituto Nacional de Estadística y Censos, se realiza la Encuesta Nacional de Salud y Nutrición (ENSANUT-ECU) la cual reporta en su último informe (13), que en la Sierra Rural la prevalencia del consumo inadecuado de proteínas $(10,9 \%)$ de hierro $(76,3 \%)$, de zinc $(26,4 \%)$, de vitamina A $(92,3 \%)$, así como del consumo excesivo de carbohidratos (39,2\%), presenta porcentajes que se encuentran sobre el promedio de la población. En dicha publicación, se advierte que debido a limitaciones de la metodología usada, "no es posible alcanzar una buena precisión"(13). Lo anterior indica que en el país se carece de información confiable sobre la ingesta habitual de energía y nutrientes a nivel de la población en general, y, en particular, de la situación existente en las zonas rurales.

Adicionalmente, la ENSANUT-ECU (13) reporta haber usado el "recordatorio de 24 horas de un solo día, empleando la metodología sugerida por Jahns et al., para ajustar por la variabilidad intraindividual" para obtener información sobre el consumo de alimentos en la población ecuatoriana, pero no proporciona detalles sobre cuáles son los datos que sirven de referencia para obtener la varianza externa estimada ni realizan discusión sobre las condiciones que hacen que la varianza externa que han tomado sea la apropiada para ajustar la distribución usual de nutrientes en la ingesta ecuatoriana. En todo caso, al momento de aplicar cualquier varianza externa estimada debe discriminarse entre centros urbanos y poblaciones rurales, tomar en cuenta los rangos de edad de los grupos y el nutriente particular de la ingesta de cuya estimación se extrajo dicha varianza.

Poseer datos sobre la ingesta habitual de los individuos y específicamente de niños y adolescentes en edad escolar, representa una información clave que permite a los estados implementar las políticas adecuadas para mejorar la salud de la población. Esto adquiere mayor relevancia en los sectores rurales, socioeconómicamente deprimidos, que históricamente han sido los más vulnerables; ya que la ingesta inadecuada de los mismos constituye un factor de riesgo que puede ocasionar la aparición de enfermedades crónicas no transmisibles.

El presente estudio, realizado en estudiantes de áreas rurales del Cantón Pujií, de la Provincia de Cotopaxi, con edades entre 8 y 15 años, tuvo por objetivo determinar: 1) Los requerimientos energéticos individuales tomando en cuenta los niveles de actividad física que realizan; 2) los niveles de suficiencia de energía y de macronutrientes (grasas, proteínas y carbohidratos), y del equilibrio de la dieta según los estándares internacionales.

\section{SUJETOS Y MÉTODOS}

La presente investigación estuvo enmarcada en el Proyecto SER MEJORES ${ }^{1}$, desarrollado en las siete instituciones educativas públicas existentes (2014-2015) en la Zona 3 de planificación, ubicada en el Cantón Pujilí, de la Provincia de Cotopaxi, que por una parte, constituye una área de influencia de la Universidad de las Fuerzas Armadas (ESPE), que fungió como institución de acogida ante la Secretaría Nacional de Educación Superior, Ciencia, Tecnología e Innovación (SENESCYT) para el desarrollo del proyecto de investigación y por otra, corresponde a una de los cantones más pobres del país (15) con $85,4 \%$ de población rural. Específicamente, las instituciones educativas participantes en el Proyecto fueron: 1) Unidad Educativa Delia Ibarra de Velasco (Barrio La Merced), 2) Colegio Nacional Manuel Eduardo Cepeda (Alpamálag de Acurios); 3) Unidad Educativa Miguel de Cervantes (Alpamálag de Acurios); 4) Escuela Básica Guaranda (Barrio Isinche de Tovares-Infantes); 5) Escuela Fiscal República de Italia (Alpamálag de Ramos Pamba); 6) Escuela Fiscal Modesto Villavicencio (Barrio Patoa de Quevedo); y la 7) Escuela Fiscal Mixta Felipe Sarrade (San José de Alpamálag).

El diseño de la investigación fue no experimental, de campo, con estudio evolutivo longitudinal y multivariable de rasgo ya que el foco de atención estuvo dirigido a varios eventos del contexto (16).

Para las mediciones del peso y la talla de los estudiantes, se siguieron los pasos especificados en el Instructivo del Ministerio de Educación ecuatoriano (17) con dos registros de cada medida. Se cumplió con los estándares éticos internacionales para la investigación con seres humanos (18), con la autorización de los padres los estudiantes participantes y con los avales de la Zonal $N^{\circ} 3$ y de la Dirección Distrital de Educación Pujilí-Saquisilí para la realización de la investigación en las instituciones educativas en las que se desarrolló el Proyecto Ser Mejores.

Como apoyo para el cálculo de los requerimientos energéticos individuales, se practicó un cuestionario abierto, tanto a los estudiantes como a sus padres, en donde se solicitó información sobre las actividades realizadas durante el día, la forma de traslado utilizada para ir a la escuela y su duración. Cruzando la información proporcionada se logró clasificar la actividad física realizada por cada uno de los estudiantes y, en los casos donde hubo discrepancia, se verificó nuevamente la información suministrada por ambas fuentes.

Para la clasificación de la actividad física realizada se utilizó el criterio de Torun (19), aceptado por la Consulta de Expertos FAO/WHO/UNU (20, p. 29-30) para grupos de poblaciones sobre los 5 años de edad, que establece que la proporción del Gasto Energético Total (GET) que corresponde a los individuos según las actividades físicas desarrolladas.

La aplicación de un par de pruebas piloto permitió establecer algunos criterios para la inclusión de los grupos participantes en la investigación, para de esta forma tener una mejor aproximación a los evento en estudio: Se realizó un muestreo por autoselección (16), conformado por secciones completas de tres grados de educación básica (quinto, sexto, séptimo) y tres de bachillerato (octavo, noveno, décimo). No se consideró la inclusión de estudiantes que cursaban grados inferiores con la finalidad de disponer de una primera fuente 
con la capacidad de suministrar datos confiables. No se incluyeron grados superiores al décimo debido a que éste constituía el último grado común en al menos dos escuelas.

Para levantar los datos de la ingesta, el trabajo de campo se desarrolló en dos etapas. En la primera, se aplicó un cuestionario abierto con Recordatorio de 24 horas a las secciones predefinidas en las instituciones educativas conformadas por 379 niños y adolescentes con edades comprendidas entre los 8 y los 15 años (edades sexo femenino: media 11,27; desviación típica 1,878; edades sexo masculino: media 10,98; desviación típica 1,829); 198 varones y 181 estudiantes de sexo femenino.

Esta primera etapa tenía la finalidad de levantar, simultáneo a la ingesta, un listado que permitiera crear una categorización general de los alimentos presentes en las comidas de los estudiantes. La aplicación del instrumento se realizó durante los días laborales de las escuelas, es decir, de lunes a viernes, con correspondencia de los datos a los días de domingo hasta el jueves. Como producto de la aplicación de este primer instrumento se identificaron 317 alimentos los cuales pudieron ser agrupados en las siguientes categorías: 1) Arroces, 2) carnes y derivados cárnicos, 3) bebidas, 4) sopas, caldos y coladas, 5) frutas, 6) hortalizas y verduras, 7) leguminosas, 8) masas y panes, 9) huevos, 10) leche y sus derivados, 11) alimentos a base de cereales y 12) golosinas y bocadillos.

La segunda etapa de la investigación se llevó a cabo en una submuestra probabilística estratificada (114 escolares) equivalente al $30 \%$ del total de estudiantes $(p=0,88$, proporción de individuos que se ajusta al esquema de categorías definidas, 95\% de nivel de confianza y un 5\% de error de muestreo). Los estratos definidos fueron a) las instituciones educativas, b) el sexo y c) la edad. En los grupos iniciales de cada escuela, una vez definido el tamaño de los estratos, se realizó una selección al azar. En este grupo se empleó un cuestionario semiestructurado en un todo de acuerdo con las categorías definidas durante la primera etapa del estudio, y se aplicó, en días no consecutivos, el método de Recordatorio de 24 Horas de Pasos Múltiples (R24HPM), adaptado a las fases del USDA Automated Multiple Pass Method creado por el United States Department of Agriculture (21-24) con registro de ingredientes de preparación y cantidades consumidas. La validez de contenido del cuestionario fue evaluada a través del juicio de 12 expertos haciendo uso del coeficiente de concordancia $W$ de Kendall, el que indica que se cumple el supuesto ( $W=0,116, g \mid=11, p>0,05)$.

Con los datos de consumo de cada uno de los recordatorios de 24 horas, se calcularon los totales correspondientes a gramos de grasa, proteína, fibra, carbohidratos y la energía consumida al día; haciendo uso de los datos presentados en diferentes tablas de composición de alimentos ecuatorianos (25-34).

La estimación de la ingesta habitual fue realizada haciendo uso del PC_SIDE (8) clasificando los datos de los estudiantes por sexo, por secuencia de aplicación del Recordatorio de 24 horas, por edad, por escuela y considerando el peso registrado en kilos.

\section{RESULTADOS}

De acuerdo a Torún (19) la actividad física encontrada en los niños y adolescentes del estrato rural resultó ser más alta que la que se podría esperar en el entorno urbano para grupos de igual edad y sexo $(35,36)$, coincidiendo además con Herrera (37) en que todos los niños de más de 10 años realizan actividades físicas, cuando menos, de intensidad moderada.

Para el cálculo de los requerimientos energéticos individuales, tomando en cuenta la talla alcanzada por cada uno de los niños y adolescentes pertenecientes al grupo de estudio y haciendo uso de las medianas proporcionadas por las tablas del Índice de Masa Corporal (IMC) de la World Health Organization (38), discriminadas por sexo y por edad, se obtuvieron los pesos correspondientes. Posteriormente, con las ecuaciones, propuestas por la consulta de expertos FAO/WHO/UNU (20), discriminadas por sexo, se calculó el GET correspondiente a la actividad física moderada, haciendo el ajuste posterior a nivel individual. Finalmente, se sumó, discriminada por sexo y edad, la energía correspondiente al crecimiento, Eg, según estimaciones de la referida consulta FAO/WHO/UNU (20). Los resultados obtenidos se presentan en las tablas 1 y 2 .

La ingesta usual de macronutrientes, específicamente de grasa, carbohidratos y proteínas, también fue obtenida haciendo uso del software PC-SIDE. No hay suficiente información científica contrastada para establecer los requerimientos promedios estimados (EAR) para macronutrientes como las grasas y los carbohidratos, sin embargo para las proteínas, en grupos de niños de ambos sexos, entre 8 y 13 años, el

TABLA 1

Requerimientos mínimos de energía estimados, sobre la base en los datos individuales, para los diferentes grupos etarios en el sexo femenino.

\begin{tabular}{|c|c|c|c|c|c|c|c|c|c|}
\hline \multirow[t]{2}{*}{ EDAD } & \multirow{2}{*}{$\begin{array}{l}\text { IMC } \\
\text { MED }\end{array}$} & \multirow{2}{*}{$\begin{array}{l}\text { MED } \\
\text { TALLA }\end{array}$} & \multirow{2}{*}{$\begin{array}{l}\text { MED } \\
\text { PESO }\end{array}$} & \multirow{2}{*}{$\begin{array}{l}\text { GET } \\
\text { MOD }^{2}\end{array}$} & \multicolumn{3}{|c|}{ ACTIVIDAD FíSICA (\%) } & \multirow[t]{2}{*}{$\mathrm{Eg}$} & \multirow{2}{*}{$\begin{array}{c}\text { Requerimiento } \\
\text { Energía total }\end{array}$} \\
\hline & & & & & LIGERA & MODERADA & INTENSA & & \\
\hline 8 & 15,9 & 1,230 & 24,1 & 1571,5 & 33,33 & 33,33 & 33,33 & 21 & 1592,3 \\
\hline 9 & 16,3 & 1,280 & 26,7 & 1683,5 & 60 & 12 & 28 & 23 & 1625,7 \\
\hline 10 & 16,9 & 1,310 & 29,0 & 1775,4 & 0 & 62,5 & 37,5 & 25 & 1900,2 \\
\hline 11 & 17,6 & 1,385 & 33,8 & 1958,9 & 0 & 72,73 & 27,27 & 25 & 2064,1 \\
\hline 12 & 18,4 & 1,413 & 36,7 & 2049,6 & 0 & 69,23 & 30,77 & 26 & 2170,2 \\
\hline 13 & 19,2 & 1,493 & 42,8 & 2226,5 & 0 & 65,22 & 34,78 & 24 & 2366,7 \\
\hline 14 & 19,9 & 1,496 & 44,5 & 2280,0 & 0 & 63,16 & 36,84 & 19 & 2425,0 \\
\hline 15 & 20,5 & 1,500 & 46,1 & 2316,6 & 0 & 62,5 & 37,7 & 12 & 2464,3 \\
\hline
\end{tabular}

2: GET correspondiente a la actividad física de intensidad moderada, es obtenido a través de las ecuaciones propuestas por la Consulta Mixta de Expertos FAO/WHO UNU (20), según la fórmula $263,4+65,3(\mathrm{Kg})-0,454\left(\mathrm{Kg}^{2)}(\mathrm{Kcal} / \mathrm{d})\right.$ para el sexo femenino. 
EAR establecido es $0,76 \mathrm{~g} / \mathrm{kg} / \mathrm{d}$; para los varones entre 14 y 18 años, de 0,73 /g/kg/d, y para las adolescentes, en este último rango de edades, 0,71 $\mathrm{g} / \mathrm{kg} / \mathrm{d}$, (39). El cálculo según los requerimientos individuales permitió establecer que todo el grupo de estudiantes se encuentra sobre el EAR para la ingesta de proteína.

Hay otros índices que resultan relevantes para evaluar la calidad de la nutrición en una población como el Rango Aceptable de Distribución de Macronutrientes (AMDR); la ingesta de grasa, oscila entre $20 \%$ y $35 \%$ de la energía $(39,40)$ donde el límite inferior está dado por la cantidad de grasas necesarias para asegurar la ingesta de ácidos grasos esenciales y de la correspondiente energía para facilitar la absorción de las vitaminas liposolubles.

Por su parte, el porcentaje-meta de carbohidratos totales, para individuos entre 14 y 18 años, oscila entre $45 \%$ y $75 \%$ del porcentaje de energía total disponible después de considerar la energía consumida en forma de proteínas y grasas. Mientras que en el caso de las proteínas, el Rango Aceptable de Distribución de Macronutrientes (AMDR) oscila entre 10\% y $30 \%$ de la energía total $(14,39)$. Para la transformación de los porcentajes de energía en gramos, se toma en cuenta el número de kcal necesarios para la combustión. $(41,42)$. La consulta de expertos de la FAO/OMS (43) coincide en que cuando la ingesta de carbohidratos supera $75 \%$ del consumo de energía total, ello puede incidir de una manera adversa en el estado de la nutrición debido a que no se estaría alcanzando el porcentaje mínimo requerido de proteínas, grasas y de otros nutrientes esenciales.

Para evaluar la ingesta de los estudiantes en relación

\section{TABLA 2}

Requerimientos mínimos de energía estimados, sobre la base en los datos individuales, para los diferentes grupos etarios en el sexo masculino.

\begin{tabular}{|c|c|c|c|c|c|c|c|c|c|}
\hline \multirow{2}{*}{ EDAD } & \multirow{2}{*}{$\begin{array}{l}\text { IMC } \\
M^{\prime} D^{3}\end{array}$} & \multirow{2}{*}{$\begin{array}{c}\text { MED } \\
\text { TALLA }^{4}\end{array}$} & \multirow{2}{*}{$\begin{array}{l}\text { MED } \\
\text { PESO }^{5}\end{array}$} & \multirow{2}{*}{$\begin{array}{c}\text { GET } \\
\text { MOD }^{6}\end{array}$} & \multicolumn{3}{|c|}{ ACTIVIDAD FÍSICA $(\%)^{7}$} & \multirow{2}{*}{$\mathrm{Eg}^{8}$} & \multirow{2}{*}{ RET $^{9}$} \\
\hline & & & & & LIGERA & MODERADA & INTENSA & & \\
\hline 8 & 15,9 & 1,260 & 25,2 & 1740,5 & 70 & 10 & 20 & 16 & 1626,0 \\
\hline 9 & 16,2 & 1,290 & 27,1 & 1825,5 & 32,5 & 22,5 & 45 & 19 & 1878,8 \\
\hline 10 & 16,7 & 1,310 & 29,0 & 1908,3 & 0 & 57,89 & 42,11 & 22 & 2050,8 \\
\hline 11 & 17,2 & 1,350 & 32,1 & 2036,0 & 0 & 76,92 & 23,08 & 25 & 2131,5 \\
\hline 12 & 17,9 & 1,400 & 36,1 & 2207,3 & 0 & 61,54 & 38,46 & 29 & 2363,6 \\
\hline 13 & 18,6 & 1,470 & 41,5 & 2441,1 & 0 & 85 & 15 & 33 & 2529,0 \\
\hline 14 & 19,4 & 1,540 & 47,2 & 2686,9 & 0 & 76,19 & 23,81 & 33 & 2815,8 \\
\hline 15 & 20,1 & 1,565 & 50,2 & 2789,0 & 0 & 66,67 & 33,33 & 30 & 2958,5 \\
\hline
\end{tabular}

3: Mediana del Índice de Masa Corporal según la World Health Organization (38).

4: Mediana correspondiente la talla en metros, según datos levantados en el Proyecto SER MEJORES; Cantón Pujilí Provincia de Cotopaxi (2014-2015).

5: Mediana del Peso $=$ Mediana $(I M C) *$ Mediana $(\text { Talla })^{2}$

6: GET moderado para el sexo femenino es obtenido a través de la fórmula: según la fórmula y 310,2 + 63,3 (Kg) - 0,263 para el sexo masculino (Kcal/d), propuesta por la FAO/WHO/UNU (20).

7: Datos obtenidos en el Proyecto SER MEJORES; Cantón Pujilí Provincia de Cotopaxi (2014-2015)

8: Eg Requerimientos de energía tisular durante el crecimiento, según Human Energy Requirements Report of a Joint FAO/WHO/UNU (20, p.27).

9: Requerimiento de energía total (RET) del grupo para una determinada edad es obtenido como la suma de los distintos requerimientos ponderados de energía más la energía requerida para el crecimiento.

\section{TABLA 3}

Distribución de porcentajes de individuos, por grupos etarios, que no alcanzan el límite inferior de la AMDR (Rango Aceptable de Distribución de Macronutrientes).

\begin{tabular}{|c|c|c|c|c|c|c|c|c|}
\hline \multirow{2}{*}{$\begin{array}{c}\text { PINALIM* } \\
(\%)\end{array}$} & \multicolumn{2}{|c|}{ ENERGÍA } & \multicolumn{2}{|c|}{ GRASA } & \multicolumn{2}{|c|}{ PROTEÍNAS } & \multicolumn{2}{|c|}{ CARBOHIDRATOS } \\
\hline & $M$ & $\mathrm{~F}$ & $M$ & $\mathrm{~F}$ & $M$ & $\mathrm{~F}$ & $M$ & $\mathrm{~F}$ \\
\hline EDAD 8 & 40,0 & 50,0 & 50,0 & 33,3 & 0,0 & 16,7 & 0,0 & 25,0 \\
\hline 9 & 47,5 & 64,0 & 55,0 & 52,0 & 20,0 & 16,0 & 10,0 & 24,0 \\
\hline 10 & 68,4 & 78,1 & 65,8 & 75,0 & 26,3 & 31,3 & 13,2 & 37,5 \\
\hline 11 & 71,8 & 75,8 & 76,9 & 75,8 & 25,6 & 39,4 & 17,9 & 36,4 \\
\hline 12 & 73,1 & 73,1 & 69,2 & 76,9 & 38,5 & 50,0 & 34,6 & 53,8 \\
\hline 13 & 100,0 & 87,0 & 85,0 & 95,7 & 50,0 & 43,5 & 30,0 & 52,2 \\
\hline 14 & 95,2 & 84,2 & 95,2 & 89,5 & 47,6 & 47,4 & 61,9 & 52,6 \\
\hline 15 & 100,0 & 100,0 & 100,0 & 100,0 & 100,0 & 75,0 & 66,7 & 62,5 \\
\hline PROMEDIO & 74,5 & 76,5 & 74,6 & 74,8 & 44,0 & 39,9 & 33,5 & 43 \\
\hline
\end{tabular}

*Prevalencia de las Ingestas que no alcanzan el límite inferior de las metas (\%). 
a los requerimientos-meta, sobre la base de los intervalos recomendados (AMR) (39) se calcularon los requerimientos mínimos de grasa, carbohidratos y proteínas, tanto a nivel individual como los correspondientes a las medianas de cada uno de los grupos discriminados por sexo y edad. Una vez obtenidas los consumos habituales a través del PC_SIDE, se procedió a calcular las prevalencias de las ingestas que no alcanzan las metas establecidas por el AMR comparando en cada caso dichas ingestas con el requerimiento individual estimado, (tablas 3 y 4).

Para determinar el nivel de equilibrio de la dieta, expresado como el porcentaje de aporte al consumo usual de energía por parte de los macronutrientes, se calcularon los promedios correspondientes para cada una de las edades, discriminando los resultados por sexo (tabla 5).

Aplicando el estadístico de Levene y la prueba F de Fisher, se aceptan las hipótesis de homogeneidad de varianzas y medias para todos los grupos etarios y se rechaza la misma para las medias de los grupos discriminados por sexo.

Para obtener la prevalencia del exceso en el consumo de grasa y de carbohidratos, se consideraron los límites máximos recomendados en la ingesta de 35\% y $75 \%$, respectivamente, en relación al requerimiento de energía calculado de forma individual en atención a las actividades físicas desarrolladas. Los resultados, discriminados por edad y por sexo, se presentan en la tabla 6.

Para los grupos clasificados de acuerdo a sexo, se realizó el análisis ANOVA de un factor usando el estadístico de Levene con su nivel de significación para contrastar la hipótesis de igualdad de varianzas de la ingesta usual de energía y de macronutrientes en los grupos clasificados a su vez por edad y por institución educativa.

El nivel crítico resultante arrojó niveles de significación mayores que 0,05 en todos los casos por lo que se concluye que no existen diferencias significativas en la ingesta usual con respecto ni a la edad ni a la institución educativa a la cual pertenecen los individuos. Para determinar el nivel de significación intraclase, se hizo uso de la prueba F de Fisher y se obtuvo nuevamente un nivel de significación superior a 0,05 por lo tanto, se acepta la hipótesis de la igualdad entre

\section{TABLA 4}

Distribución de porcentajes del requerimiento cubierto por la ingesta usual en individuos que no alcanzan el límite inferior de la AMDR (Rango Aceptable de Distribución de Macronutrientes).

\begin{tabular}{|c|c|c|c|c|c|c|c|c|c|}
\hline \multicolumn{2}{|c|}{ \%RIU-INCRM* } & \multicolumn{2}{|c|}{ ENERGÍA } & \multicolumn{2}{|c|}{ GRASA } & \multicolumn{2}{|c|}{ PROTEÍNAS } & \multicolumn{2}{|c|}{ CARBOHIDRATOS } \\
\hline & & $M$ & $\mathrm{~F}$ & $M$ & $\mathrm{~F}$ & $M$ & $\mathrm{~F}$ & $M$ & $\mathrm{~F}$ \\
\hline \multirow[t]{8}{*}{ EDAD } & 8 & 96,5 & 71,9 & 82,9 & 70,0 & - & 75,4 & - & 80,5 \\
\hline & 9 & 79,2 & 75,8 & 71,9 & 69,7 & 83,1 & 72,3 & 80,5 & 85,2 \\
\hline & 10 & 82,1 & 70,7 & 72,6 & 71,9 & 87,2 & 82,6 & 91,9 & 81,2 \\
\hline & 11 & 79,3 & 68,0 & 73,4 & 73,9 & 89,9 & 79,0 & 83,2 & 72,2 \\
\hline & 12 & 70,8 & 62,5 & 63,5 & 68,5 & 81,6 & 79,8 & 87,9 & 80,6 \\
\hline & 13 & 73,5 & 63,1 & 64,3 & 66,5 & 85,0 & 74,1 & 83,7 & 73,3 \\
\hline & 14 & 67,5 & 59,0 & 63,8 & 65,3 & 68,6 & 68,2 & 86,4 & 63,7 \\
\hline & 15 & 65,7 & 64,9 & 67,3 & 67,5 & 92,8 & 84,9 & 90,0 & 74,2 \\
\hline \multicolumn{2}{|c|}{ PROMEDIO } & 76,8 & 67,0 & 70,0 & 69,2 & 84,0 & 77,0 & 86,2 & 76,4 \\
\hline
\end{tabular}

*Porcentaje del requerimiento cubierto por la ingesta usual (\%) en individuos que no cumplen requerimiento-meta.

TABLA 5

Distribución porcentual del aporte de los macronutrientes a la ingesta usual de energía.

\begin{tabular}{|c|c|c|c|c|c|c|c|c|c|}
\hline \multirow{2}{*}{\multicolumn{2}{|c|}{$\begin{array}{c}\text { ACTE* }^{*} \\
(\%)\end{array}$}} & \multicolumn{2}{|c|}{$\begin{array}{c}\text { ENERGÍA } \\
\text { Ingesta usual }\end{array}$} & \multicolumn{2}{|c|}{$\begin{array}{c}\text { GRASA } \\
(20-35 \%)\end{array}$} & \multicolumn{2}{|c|}{$\begin{array}{c}\text { PROTEÍNAS } \\
(10-30 \%)\end{array}$} & \multicolumn{2}{|c|}{$\begin{array}{c}\text { CARBOHIDRATOS } \\
(45-65 \%)\end{array}$} \\
\hline & & $M$ & $F$ & $M$ & $F$ & $M$ & $\mathrm{~F}$ & $M$ & $\mathrm{~F}$ \\
\hline \multirow[t]{8}{*}{ EDAD } & 8 & 1972,8 & 1555,7 & 21,7 & 22,2 & 14,8 & 15,8 & 64,5 & 63,5 \\
\hline & 9 & 2046,1 & 1665,6 & 20,4 & 21,2 & 15,1 & 15,1 & 69,1 & 65,8 \\
\hline & 10 & 1904,5 & 1603,1 & 19,7 & 21,3 & 14,6 & 15,2 & 64,9 & 64,0 \\
\hline & 11 & 1934,2 & 1681,0 & 20,4 & 21,5 & 14,7 & 15,2 & 65,3 & 63,9 \\
\hline & 12 & 2066,4 & 1717,8 & 19,8 & 21,6 & 14,9 & 15,1 & 65,2 & 64,1 \\
\hline & 13 & 1829,7 & 1652,5 & 19,9 & 21,7 & 14,2 & 15,4 & 66,1 & 64,6 \\
\hline & 14 & 1925,3 & 1717,1 & 18,6 & 21,2 & 13,9 & 15,2 & 67,6 & 64,9 \\
\hline & 15 & 1878,8 & 1571,2 & 20,6 & 21,4 & 14,2 & 15,1 & 65,2 & 64,4 \\
\hline \multicolumn{2}{|c|}{ PROMEDIO } & 1944,7 & 1645,5 & 20,1 & 21,5 & 14,6 & 15,3 & 66,0 & 64,4 \\
\hline
\end{tabular}

*Aporte al consumo total de energía. 
las medias, esto indica que ni la edad ni la institución educativa de procedencia producen efectos distintos ni en la ingesta usual de la energía ni en la de macronutrientes; y este comportamiento fue idéntico en ambos sexos. Sin embargo, en los grupos clasificados de acuerdo al sexo, el ANOVA intergrupos resultó ser significativo. Las características de la ingesta usual, obtenida haciendo uso del PC-SIDE, se presentan en la tabla 7.

\section{DISCUSIÓN}

Las prevalencias de las ingestas que no satisfacen los requerimientos de energía y las metas establecidas para la ingesta de macronutrientes se incrementan progresivamente con la edad, ello se explica porque las cantidades de alimentos consumidas en general permanecen constantes, pero los requerimientos se hacen mayores producto del desarrollo físico.

La ingesta promedio, en ambos sexos, se encuentra por debajo de los requerimientos necesarios estimados para las actividades físicas desarrolladas, siendo más marcada en el sexo femenino. La estimación del porcentaje promedio del requerimiento de energía que es cubierto por la ingesta usual de los grupos, es mayor en los varones, alcanzando $88,5 \%$ contra $81,8 \%$ en las niñas.

En ambos sexos, los porcentajes de grasa y de proteínas aportados en relación a la energía total, se encuentran dentro del rango recomendado, inclinándose ambos al extremo inferior y resultando más altos en el sexo femenino que en el masculino. En cuanto a la ingesta de carbohidratos se encontró que el porcentaje promedio de aporte se encuentra en el extremo superior siendo más alto en los varones.

El estudio individual de los datos (tablas 3 y 4), revela que algunos escolares a pesar de no cumplir con las metas establecidas para el consumo de energía, grasa, proteína o carbohidratos, satisfacen con la ingesta usual un porcentaje de esos requerimientos que, en promedio, es superior al $70 \%$ en el caso de la energía y de grasa, y por sobre el $80 \%$ en las proteínas y los carbohidratos. Los porcentajes promedios de requerimientos cubiertos por la ingesta usual son mayores en los varones ya que presentan una mayor ingesta que las niñas.

Entre los 8 y 9 años, se presenta la menor prevalencia de ingestas que no cumplen con las metas establecidas por la Consulta Mixta de Expertos OMS/FAO/UNU, siendo en la energía y la grasa inferior al 55\% y en las proteínas y carbohidratos, menor del $25 \%$. También corresponde a este rango de edades el mayor porcentaje de requerimiento cubierto por la ingesta usual, llegando a alcanzar, en el caso de las proteínas y de las grasas, un exceso que oscila entre 39, 1 y $84,1 \%$. En el otro extremo del rango de edades estudiado, entre los 14 y los 15 años, en el caso de la energía y la grasa, el porcentaje de las metas propuestas que logra cubrirse oscila entre $64,8 \%$ y $71,7 \%$.

Se observó una tendencia de los escolares con edades comprendidas entre los 8 y 9 años a presentar un consumo excesivo de carbohidratos (aproximadamente 37\% de exceso en las niñas y más del $70 \%$ en los varones) cuya fuente principal es el arroz y la papa, este comportamiento se anula completamente en el grupo de adolescentes de 15 años en los que se manifiesta una mayor selección de los alimentos que consumen, (tabla 6).

En esta investigación se encontró que la totalidad de los escolares cumple el EAR, lo cual encuentra justificación si se toman en cuenta, dos factores: 1) La existencia del Programa de Alimentación Escolar que aporta de un desayuno diario a los estudiantes que contiene entre 12 y 14 gramos de proteína lo cual constituye un aporte promedio, en ambos sexos, de

\section{TABLA 6}

Distribución porcentual de estudiantes, por grupos etarios, que presentan exceso en el consumo de grasa y carbohidratos.

\begin{tabular}{ccccc}
\hline (\%) & \multicolumn{2}{c}{$\begin{array}{c}\text { Exceso en consumo de grasa } \\
\text { FEMENINO }\end{array}$} & MASCULINO & $\begin{array}{c}\text { Exceso en consumo de carbohidratos } \\
\text { FEMENINO }\end{array}$ \\
Edad & 8 & 0 & 30 & 41,790 \\
9 & 16 & 7,5 & 32 & 55 \\
10 & 3,1 & 2,6 & 18,8 & 28,9 \\
11 & 3,0 & 5,1 & 21,2 & 33,3 \\
12 & 0 & 7,7 & 23,1 & 23,1 \\
13 & 0 & 0 & 17,4 & 0 \\
14 & 0 & 0 & 15,8 & 0 \\
15 & 0 & 0 & 0 & 0 \\
\hline
\end{tabular}

TABLA 7

Características de la ingesta usual.

\begin{tabular}{lcc}
\hline INGESTA USUAL & MASCULINO & FEMENINO \\
ENERGÍA (Kcal) & $1937,3 \pm 538,3$ & $1657,1 \pm 634,9$ \\
GRASA (g) & $42,7 \pm 18,7$ & $38,2 \pm 14,8$ \\
PROTEÍNAS (g) & $70,1 \pm 24,0$ & $61,8 \pm 23,3$ \\
CARBOHIDRATOS (g) & $314,8 \pm 83,3$ & $264,3 \pm 105,0$ \\
\hline
\end{tabular}


$50 \%$ del EAR de proteína para los niños y de casi 35\% para los adolescentes; y 2) la cantidad y variedad de leguminosas que están presentes en las dietas de los escolares del Cantón Pujilí.

En promedio, la ingesta usual de $75 \%$ de los estudiantes no cumple ni con los requerimientos de energía según la actividad física realizada ni con la meta establecida por la Consulta Mixta de Expertos para el consumo de grasa, y $40 \%$ no cumple el requisito-meta de proteínas y carbohidratos; sin embargo, este grupo de estudiantes cubre con su ingesta más de un $70 \%$ de los requerimientos energéticos y entre un 69,2 y un $86,2 \%$ de las metas fijadas para el consumo de macronutrientes. En todos los casos, se encontró que la ingesta usual de los varones se acerca en mayor porcentaje a las metas, ello tiene explicación en la tendencia socio-cultural de los estratos rurales de la Sierra Ecuatoriana en donde se privilegia la alimentación de los varones.

\section{CONCLUSIONES}

La investigación realizada permitió estimar los requisitos de energía en grupos de escolares de estratos rurales de la Sierra Ecuatoriana, con edades entre 8 y 15 años, por sexo y en atención a las actividades físicas que realizan.

A pesar de que la prevalencia de ingestas que no alcanzan los requerimientos energéticos estimados ni las metas establecidas (39) para el consumo de grasa, se ubica cerca del $75 \%$ para ambos sexos, dicha meta es cubierta aproximadamente en $70 \%$ por esos estudiantes cuyo consumo habitual está por debajo de la ingesta deseable. En el caso de las proteínas y los carbohidratos, el porcentaje de estudiantes que no alcanza el Rango Aceptable de Distribución de Macronutrientes (AMDR), es aproximadamente de $40 \%$; sin embargo, éste es cubierto en más de $80 \%$ por la ingesta usual.

El $100 \%$ de los estudiantes participantes en el proyecto, cumple con el Requerimiento Promedio Estimado (EAR) para la proteína.

El mayor exceso de consumo de carbohidratos se encontró en los estudiantes más jóvenes.

El aporte porcentual del consumo estimado de grasas, proteínas y carbohidratos, respecto a de la ingesta usual de la energía, se encuentra enmarcado en los requisitos-meta establecidos (39).

\section{RESUMEN}

El presente estudio, se realizó en estudiantes de áreas rurales del Cantón Pujilí, Provincia Cotopaxi, con edades entre 8 y 15 años, el que permitió determinar: 1) Los requerimientos energéticos individuales según los niveles de actividad física realizada; 2) los niveles de suficiencia de energía y macronutrientes y de equilibrio de la dieta a la luz de los estándares internacionales. Se encontró que más del 70\% de los escolares no cumplían las metas fijadas por la Consulta Mixta de Expertos (Organización Mundial de la Salud/ Organización de las Naciones Unidas para la Agricultura y la Alimentación/ Universidad de las Naciones Unidas). En relación al consumo de energía y grasas; dicho porcentaje fue inferior al $40 \%$ en el caso de las proteínas y los carbohidratos; y que el aporte porcentual de la ingesta usual de macronutrientes respecto al consumo estimado de energía se encuentra enmarcado en los respectivos requerimientos-meta establecidos.

Palabras clave: Ingesta usual; requerimientos energéticos; macronutrientes; equilibrio de la dieta; escuelas rurales.

Agradecimientos: El presente trabajo fue patrocinado por el Proyecto Prometeo de la Secretaría de Educación Superior,
Ciencia, Tecnología e Innovación de la República del Ecuador, SENESCYT, a quiénes agradezco por hacer posible la realización de la investigación.

De igual forma se agradece a la Coordinadora de Prometeros-ESPE, Ing. Patricia Falconí, al Director del Departamento de Ciencias Humanas y Sociales, CRNL Marco Ayala y a los docentes de la Carrera de Lingüística de la ESPE, especialmente al Máster Néstor Bonilla, por toda la colaboración prestada para el levantamiento de la información.

De manera particular, hago un reconocimiento a mi hija, Mailevy Fabiana Guía Pereira, por todo el apoyo brindado a lo largo de esta investigación. A todos, mil gracias.

\section{BIBLIOGRAFÍA}

1. Organización Mundial de la Salud. Nutrición. [Portal en línea] Disponible: http://www.who.int/topics/nutrition/ es/ [Consulta: 2015, julio 10].

2. Ziegler E.E y L.J. Filer Jr. (Editores). Conocimientos actuales sobre nutrición (7a. ed.). Internacional Life Sciences Institute, Washington, DC, 1998, p.533.

3. Willet W. Nutr. Epidemiol. (3rd. ed.). Oxford University Press, Inc., New York, 2013.

4. Gibson RS. Principles of Nutritional Assessment (2th. ed.). Oxford University Press, Inc, New York, 2005.

5. Gil, A. Tratado de Nutrición (t.II) (2a. ed.). Médica Panamericana, Madrid, 2010.

6. Carriquiry AL. Estimation of Usual Intake Distributions of Nutrients and Foods. Department of Statistics, lowa State University, Ames: lowa, 2003, p.602.

7. National Research Council Nutrient Adequacy: Assessment Using Food Consumption Surveys. National Academy Press, Washington, DC, 1986.

8. Department of Statistics The Center for Agricultural and Rural Development. PC_SIDE Version 1.0, Copyright 2003, Buil Date: Jun 19 2013. Iowa State University, Ames: lowa, 2013.

9. Institute of Medicine. Food and Nutrition Board. Dietary Reference Intakes. Applications in Dietary Assessment. National Academy Press, Washington, DC, 2001.

10. Nusser SM, Carriquiry AL, Dodd KW, Fuller W A, Jensen HH. A User's Guide to C-SIDE (Software for Intake Distribution Estimation). Version 1.0. Dietary Assessment Research Series Report 8, A. Center for Agricultural and Rural Development, lowa State University, Ames: Iowa, 1996, p. 53.

11. Dodd, KW. A Technical Guide to C-SIDE. Software for Intake Distribution Estimation. Center for Agricultural and Rural Development, lowa State University, Ames, lowa, 1996.

12. United States Department of Agriculture. Interactive DRI Glosarry [Glosario en línea]. Disponible: https://fnic. nal.usda.gov/interactive-dri-glossary [Consulta: 2015, Agosto 13]

13. Freire $W B$, Ramírez $M J$, Belmont $P$, Mendieta, $M J$, Silva MK, Romero N, Sáenz K, Piñeiros P, Gómez LFy Monge E. Resumen Ejecutivo. Tomo I. Encuesta Nacional de Salud y Nutrición del Ecuador. ENSANUT-ECU 2011-2013. Ministerio de Salud Pública/ Instituto Nacional de Salud Pública y Censo, Quito, 2013, pp.47-50.

14. Organización Mundial de la Salud. Dieta, Nutrición y Prevención de Enfermedades Crónicas. OMS, Serie de Informes Técnicos, 916. OMS/FAO, Ginebra, 2003, p.63.

15. Ecuador Inmediato. LA GACETA (Latacunga) Pujilí entre los cantones más pobres del país [Información en línea]. 
Disponible: http://www.ecuadorinmediato.com/Noticias/ news_user_view/ ecuadorinmediato_noticias--24297 Cotopaxi, 2016 [Consulta: 2016, Enero 10]

16. Hurtado de Barrera. Metodología de la Investigación: Guía para la Comprensión Holística de la Ciencia. 4a ed. Quirón Ediciones, Caracas, 2010.

17. Ministerio de Educación. Instructivo del sistema de control del crecimiento y estado nutricional de los niños de Educación Inicial (Sicene). Ministerio de Educación del Ecuador, Quito, 2013.

18. Consejo de Organizaciones Internacionales de las Ciencias Médicas Organización Mundial de la Salud Pautas Éticas Internacionales para la Investigación Biomédica en Seres Humanos. CIOMS/OMS, Ginebra, 2002.

19. Torun B. (2001). Energy requirements of children and adolescents. Background paper prepared for the joint FAO/ WHO/UNU Expert Consultation on Energy in Human Nutrition, Public Health Nutrition, Cambridge, 2001, p.985.

20. FAO/WHO/UNU. Human Energy Requirements. Food and Nutrition Technical Report Series. Report of a Joint FAO/ WHO/UNU. Expert Consultation. Rome, 2001.

21. U.S. Department of Agriculture, Agricultural Research Service, Beltsville Human Nutrition Research Center, Food Surveys Research Group (Beltsville, MD) and U.S. Department of Health and Human Services, Centers for Disease Control and Prevention, National Center for Health Statistics (Hyattsville, MD). What We Eat in America. AMPM Features, Maryland, 2014.

22. Bliss RM. Researchers Produce Innovation in Dietary Recall. Agricultural Research, U.S. Department of Agriculture, Beltsville: Maryland, 2004; 52(6):10-12.

23. Raper $N$, Perloff $B$, Ingwersen $L$, Steinfeldt $L$ y Anand J. An overview of USDA's Dietary Intake Data System. Food Composition and Analysis, Beltsville: USA, 2004; 17(3-4):545-55.

24. McBride J. Was it a slab, a slice, or a sliver? High-tech innovations take food survey to new level. Agricultural Research, U.S. Department of Agriculture, Beltsville: Maryland, 2001: 49(3):4-7.

25. Ministerio de Previsión Social y Sanidad. Tabla de Composición de los Alimentos Ecuatorianos. Instituto Nacional de Nutrición, Quito, 1965.

26. International Network of Food Data Systems. Latin American food composition tables [Información en línea]. Food and Agriculture Organization of the United Nations. Disponible: http://www.fao.org/infoods/infoods/tablesand-databases/latin-america/en/2015, [Consulta: 2015, enero 25].

27. Instituto de Ciencias Naturales de la Universidad Central. Tabla de composición de alimentos para Ecuador. Universidad Central, Quito, 1982.

28. Red Latinoamericana de Composición de Alimentos. Tabla de composición de alimentos de América Latina [Página web en línea]. Disponible: http://www.inta.cl/latinfoods/ 2015, [Consulta: 2015, Febrero 22: 10:32]

29. FAO/INFOODS. Bases de datos FAO/INFOODS de composición de alimentos [Información en línea]. Organización de las Naciones Unidad para la Alimentación y la Agricultura Disponible: http://www.fao.org/infoods/infoods/ tablas-y-bases-de-datos/bases-de-datos-faoinfoods-decomposicion-de-alimentos/es/2013, [Consulta: 2015 enero 28: 17:29].

30. Cotier JP. Punto de vista de la FAO sobre las actividades internacionales relativas a la composición química de alimentos. En Producción y Manejo de Datos de Composición Química de Alimentos en Nutrición Editado por Cecilio Morón, Isabel Zacarías, Saturnino de Pablo. Organización de las Naciones Unidas para la Agricultura y la Alimentación, (FAO), Santiago: Chile, 1997.

31. Paucar Ñacata, DJ. (2014). Estudio Investigativo del Morocho y su Aplicación Gastronomica. Tesis presentada como requisito para optar al título de Administrador Gastronómico. Universidad Tecnológica Equinoccial, Quito, 2014.

32. Soto Enriquez R y Vilcapoma Vilcapoma DS. Evaluación de vida útil en anaquel de tres variedades de maíz (Zea Mays L.) nativo tostado y envasado en tres tipos de envases. Tesis presentada como requisito para optar al Título de Ingeniero en Industrias Alimentarias. Universidad Nacional del Centro del Perú, Huancayo, Perú, 2012.

33. International Fund for Agricultural Development. Chochos en su punto. En Programa Nacional de Leguminosas y Granos Andinos. Publicación Miscelánea N¹8. : Instituto Nacional Autónomo de Investigaciones Agropecuarias, Estación Experimental Santa Catalina, Quito, 2003.

34. Álvarez Carita, Y. C. Elaboración y caracterización de dos bebidas proteicas, una base de quínua malteada y la otra a base de quínua sin maltear (Chenopodium quinoa). Facultad de Ciencias Agropecuarias, Universidad Nacional Jorge Basadre Grohmann, Tacna: Perú, 2012.

35. Ministerio de Salud. Requerimientos de Energía para la Población Peruana. Dirección Ejecutiva de Prevención de Riesgo y Daño Nutricional. Área de Desarrollo de Recursos Humanos y Transferencia Tecnológica, Lima, 2012.

36. Comisión Económica para América Latina y el Caribe Principios y aplicación de las nuevas necesidades de energía según el Comité de Expertos FAO/OMS 2004. Estudios estadísticos y prospectivos, Serie 56. Naciones Unidas, Santiago de Chile, 2007.

37. Herrera J. Nuevas Estimaciones de la Pobreza en el Perú, 1997-2000. Instituto Nacional de Estadística e Informática, Consorcio de Investigación Económica y Social, Lima, 2001.

38. World Health Organization. Growth reference data for 1519 years, 2007. United Nations, New York, 2007.

39. Institute of Medicine, Standing Committee on the Scientific Evaluation of Dietary Reference Intakes and Food and Nutrition Board. Dietary Reference Intakes for Energy, Carbohydrate, Fiber, Fat, Fatty Acids, Cholesterol, Protein and Amino Acids. National Academies Press, Washington DC, 2002

40. Fundación Iberoamericana de Nutrición y la Organización de las Naciones Unidas para la Alimentación y la Agricultura. Grasas y Ácidos Grasos en nutrición Humana. Consulta de Expertos. Estudio FAO Alimentación y Nutrición, 91. Organización de las Naciones Unidas para la Alimentación y la Agricultura, Granada: España, 2012, p.59.

41. Marín Rodríguez ZR. Elementos de Nutrición Humana. Universidad Estatal a Distancia, San José: Costa Rica, 2014, p. 49.

42. Latham, MC. Nutrición Humana en el Mundo en Desarrollo. Colección FAO: Alimentación y nutrición, 29, Roma, 2002, p. 101.

43. FAO/OMS. Los Carbohidratos en la Nutrición Humana. Informe de una Consulta Mixta FAO/OMS de Expertos, 66, Roma, 1997, p.17. 